\title{
Thermal effects in low-temperature QED
}

\author{
Jens O. Andersen \\ Institute for Theoretical Physics, University of Utrecht, Leuvenlaan 4, 3584 CE Utrecht, The Netherlands
}

(Received 15 August 2001; published 26 December 2001)

\begin{abstract}
QED is studied at low temperature ( $T \ll m$, where $m$ is the electron mass) and zero chemical potential. By integrating out the electron field and the nonzero bosonic Matsubara modes, we construct an effective threedimensional field theory that is valid at distances $R \gg 1 / T$. As applications, we reproduce the ring-improved free energy and calculate the Debye mass to order $e^{5}$.
\end{abstract}

DOI: $10.1103 /$ PhysRevD.65.025014

PACS number(s): 12.20.Ds, 11.10.Wx

\section{INTRODUCTION}

If we have a quantum field theory in equilibrium at temperature $T$, the abundance of particles of mass $m$ much larger than $T$ is Boltzmann suppressed. More surprisingly, perhaps, is the fact that there are additional effects that are suppressed only by powers of $T / m$ [1-3]. The Boltzmann-suppressed terms can be associated with loop integrations that involve distribution functions of the heavy particle. On the other hand, the power-suppressed terms arise from perturbative corrections involving only distribution functions of light particles, with masses on the order of $T$ or less.

Effective field theory ideas suggest that one integrates out the heavy particle of mass $m$ to construct a low-energy effective field theory that can be used for $T \ll m[4,5]$. Such an approach was used by Kong and Ravndal [2] to study QED at low temperature (see also Refs. [6-13] and references therein for various calculations in low-temperature QED). By integrating out the electron field, they constructed a lowenergy effective field theory for photons. Since this procedure was carried out at zero temperature, dimensional analysis tells us that the coefficients in the effective theory are suppressed by inverse powers of the electron mass $m$. These higher order interactions are induced by the coupling of the photon to virtual electron-positron pairs in the vacuum. Since the momenta of the photons are on the order $T$ and thus much smaller than $m$, the electron-positron pairs are off their mass shell by an amount $\sim m$. Thus they can only propagate a distance $R \sim 1 / m$ and their effects can be mimicked by local interactions. Using this effective field theory to calculate corrections to the Stefan-Boltzmann law for the pressure, they showed that the leading correction varies as $\alpha^{2} T^{8} / \mathrm{m}^{4}$. This correction was obtained by a straightforward two-loop calculation in the effective theory. In full QED, it would require a three-loop calculation.

If we are interested only in power corrections, we can determine the coefficients in the effective field theory by matching at zero temperature. In the case of QED, this would lead to the Euler-Heisenberg Lagrangian $[14,15]$ with additional higher order operators that can be written in terms of the field strength and its dual [2] (see e.g. [16] for such higher order operators). However, it can be shown that this effective Lagrangian leads to a vanishing Debye mass to all orders in perturbation theory. We know that this is incorrect, but one can account for Debye screening and other Boltzmann-suppressed effects in low-temperature QED by carrying out the matching at finite temperature. Alternatively, one may reorganize the usual perturbative series in QED using resummed propagators in the usual way. However, such an approach is normally more cumbersome in practical calculations than effective field theory.

If we are interested in static quantities such as the pressure or Debye mass, it proves useful to construct a second effective field theory for the zero Matsubara mode and this is done by integrating out the nonzero Matsubara modes [1720]. This effective field theory is three dimensional and is valid at distances $R \gg 1 / T$. It can be constructed in a two-step process by first integrating out the electron field, and then integrating out the nonzero bosonic Matsubara modes. From a calculational point of view, however, it is easier to integrate out the fermions and the nonzero Matsubara modes at the same time. In this paper, we will take the latter approach.

The paper is organized as follows. In Sec. II, we discuss QED at low temperature and the construction of the threedimensional effective field theory. In Sec. III, we determine the coefficients in the effective field theory. In Sec. IV, we apply the effective field theory to calculate the free energy to order $e^{3}$ and the Debye mass to order $e^{5}$. Finally, in Sec. V, we summarize and conclude. All necessary details are collected in three Appendixes.

\section{QED AT LOW TEMPERATURE}

In the imaginary-time formalism, one can view a quantum field theory in four dimensions as a field theory in three Euclidean dimensions with an infinite tower of fields, where the Matsubara frequencies act as masses in the propagators [17]. In low-temperature QED, this implies that the fermions have masses of order $m$, while the nonzero bosonic modes have masses of order $T$. The zero-frequency bosonic modes are massless. Thus for distances $R \gg 1 / T$, we can construct an effective three-dimensional field theory for the zero Matsubara modes by integrating out the electron field as well as the nonzero bosonic modes [17-20]. The coefficients of this effective field theory then encode the physics at the momentum scales $m$ and $T$. This procedure is briefly explained in Sec. III.

The partition function of QED can be written as a path integral:

$$
\mathcal{Z}=\int \mathcal{D} \bar{\eta} \mathcal{D} \eta \mathcal{D} A_{\mu} \mathcal{D} \bar{\psi} \mathcal{D} \psi \exp \left[-\int_{0}^{\beta} d \tau \int d^{3} x \mathcal{L}\right],
$$


where the Euclidean Lagrangian is

$$
\begin{aligned}
\mathcal{L}_{E}= & \frac{1}{4} F_{\mu \nu} F_{\mu \nu}+m \bar{\psi} \psi+\bar{\psi} \gamma_{\mu}\left(\partial_{\mu}-i e A_{\mu}\right) \psi \\
& +\left(\partial_{\mu} \bar{\eta}\right)\left(\partial_{\mu} \eta\right)+\mathcal{L}_{\mathrm{GF}} .
\end{aligned}
$$

Here, $\mathcal{L}_{\mathrm{GF}}$ denotes the gauge-fixing term. In the following we work in the Feynman gauge, where

$$
\mathcal{L}_{\mathrm{GF}}=\frac{1}{2}\left(\partial_{\mu} A_{\mu}\right)^{2} .
$$

However, we emphasize that physical quantities are independent of the gauge-fixing condition.

In the three-dimensional effective theory, we can write the partition function as

$$
\mathcal{Z}=e^{-f(\Lambda) V} \int \mathcal{D} \bar{\eta} \mathcal{D} \eta \mathcal{D} \bar{A}_{0} \mathcal{D} \bar{A}_{i} \exp \left[-\int d^{3} x \mathcal{L}_{\text {eff }}\right],
$$

where the prefactor $f(\Lambda)$ is interpreted as the coefficient of the unit operator in the effective three-dimensional field theory. It depends on an ultraviolet cutoff $\Lambda$ that cancels the cutoff dependence in the path integral in Eq. (4) [19]. $\mathcal{L}_{\text {eff }}$ is the Lagrangian of the effective three-dimensional field theory. The effective field theory consists of a gauge field $\bar{A}_{i}$ coupled to a real massive self-interacting scalar field $\bar{A}_{0}{ }^{1}$ These fields can up to normalizations be identified with the zero-frequency modes of the gauge field in QED. We can then schematically write

$$
\begin{aligned}
\mathcal{L}_{\text {eff }}= & \frac{1}{4} F_{i j} F_{i j}+\frac{1}{4} a_{3}(\Lambda) F_{i j} \nabla^{2} F_{i j}+\frac{1}{2}\left(\partial_{i} \bar{A}_{0}\right)^{2}+\frac{1}{2} M^{2}(\Lambda) \bar{A}_{0}^{2} \\
& +\frac{\lambda_{3}(\Lambda)}{24} \bar{A}_{0}^{4}+\left(\partial_{i} \bar{\eta}\right)\left(\partial_{i} \eta\right)+\mathcal{L}_{\mathrm{GF}}+\delta \mathcal{L}_{\text {eff }}
\end{aligned}
$$

where $\delta \mathcal{L}_{\text {eff }}$ represents all higher order local terms that can be constructed out of $\bar{A}_{i}$ and $\bar{A}_{0}$ and that respect the symmetries of the theory. Examples of such symmetries are threedimensional gauge invariance and rotational symmetry. This includes renormalizable terms such as $F_{i j}^{2}$ as well as nonrenormalizable terms such as $\bar{A}_{0}^{6}$. Note also that we have suppressed the $\Lambda$ dependence of the fields $\bar{A}_{i}$ and $\bar{A}_{0}$ in Eq. (5). Let us finally look at the power-counting rules for the effective theory Eq. (5). The coefficients of the operators are power series in $e^{2}$ since we are ignoring infrared divergences and are using conventional perturbation theory to determine them (see Sec. III). Physical quantities are expressed in powers of the parameters $f(\Lambda), a_{3}(\Lambda), M(\Lambda)$, etc., and we must figure out at what order the operators that multiply them start to contribute. Each power of momentum in a loop integral in the effective theory gives a factor of $M$; in particular, the measure gives a factor $M^{3}$. If we want to calculate the free

\footnotetext{
${ }^{1}$ The fact that we must allow for terms such as $(1 / 2) M^{2}(\Lambda) \bar{A}_{0}^{2}$ and $\left[\lambda_{3}(\Lambda) / 24\right] \bar{A}_{0}^{4}$ is a direct consequence of the breakdown of Lorentz invariance at finite temperature.
}

energy to order $e^{3}$, it is necessary to determine $f(\Lambda)$ to order $e^{2}$. Moreover, the one-loop contribution to the free energy in the effective theory is proportional to $M^{3}$, and we therefore need to know the mass parameter to order $e$. (Note that the operator $F_{i j} \nabla^{2} F_{i j}$ does not contribute to the free energy at order $e^{2}$ since it involves only massless fields whose loop integral vanishes in dimensional regularization. In fact, this operator can be transformed away by a field redefinition at the expense of modifying the coefficients of higher order operators.) On the other hand, the operator $\bar{A}_{0}^{4}$ starts to contribute first at order $e^{6}$; its coefficient $\lambda_{3}(\Lambda)$ varies as $e^{4}$ and it gives rise to a two-loop diagram in the effective theory where each loop is proportional to $M$. In this manner we can determine at what order in $e$ an operator starts to contribute to a given physical quantity, and in Eq. (5) we have explicitly displayed those operators needed to determine the free energy to order $e^{3}$ and the Debye mass to order $e^{5}$.

\section{SHORT-DISTANCE COEFFICIENTS}

In this section, we determine the short-distance coefficients in the effective Lagrangian Eq. (5). These coefficients must be tuned as functions of $e, T$, and the ultraviolet cutoff $\Lambda$ so that the effective theory reproduces correlation functions at distances much larger than $1 / T$. We can carry out these calculations using conventional perturbation theory, which is an expansion in powers of $e^{2}$. This expansion is plagued with infrared divergences due to long-range forces mediated by the massless photon. These divergences are Debye screened, but can only be taken into account by resummation. Although the naive perturbative expansion breaks down due to these infrared divergences, it can still be used to determine the short-distance coefficients [19]. As long as we treat the long-distance physics in the same incorrect way using the effective theory, the infrared divergences will cancel in the matching equations and the coefficients properly encode the short-distance physics [19]. The Lagrangian of QED is split in the usual way into free and interacting pieces

$$
\begin{aligned}
& \mathcal{L}_{E}^{\text {free }}=\frac{1}{4} F_{\mu \nu} F_{\mu \nu}+\bar{\psi}(m+\not) \psi+\left(\partial_{\mu} \bar{\eta}\right)\left(\partial_{\mu} \eta\right)+\mathcal{L}_{\mathrm{GF}}, \\
& \mathcal{L}_{E}^{\mathrm{int}}=-i e A \bar{\psi} \psi,
\end{aligned}
$$

while the Lagrangian Eq. (5) is split according to

$$
\begin{aligned}
\mathcal{L}_{\text {eff }}^{\text {free }}= & \frac{1}{4} F_{i j} F_{i j}+\frac{1}{2}\left(\partial_{i} \bar{A}_{0}\right)^{2}+\left(\partial_{\mu} \bar{\eta}\right)\left(\partial_{\mu} \eta\right)+\mathcal{L}_{\mathrm{GF}}, \\
\mathcal{L}_{\text {eff }}^{\text {int }}= & \frac{1}{4} a_{3}(\Lambda) F_{i j} \nabla^{2} F_{i j}+\frac{1}{2} M^{2}(\Lambda) \bar{A}_{0}^{2} \\
& +\frac{\lambda_{3}(\Lambda)}{24} \bar{A}_{0}^{4}+\delta \mathcal{L}_{\text {eff }} .
\end{aligned}
$$



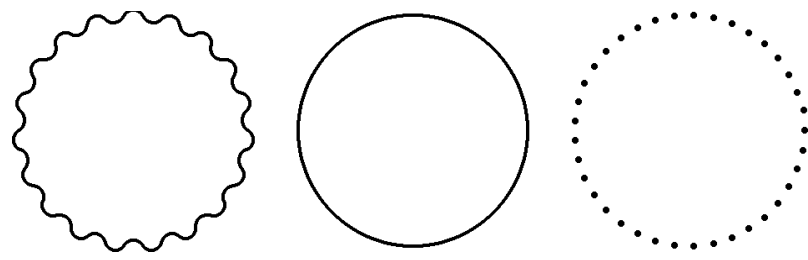

FIG. 1. One-loop vacuum diagrams in QED.

\section{A. Coefficient of the unit operator}

The matching condition that determines the coefficient of the unit operator is [19]

$$
\log \mathcal{Z}=-f(\Lambda) V+\log \mathcal{Z}_{\text {eff }} .
$$

The contributions to $\log \mathcal{Z}$ through order $e^{2}$ are given by the Feynman graphs in Figs. 1 and 2. A wavy line denotes a photon, a solid line denotes a fermion, and a dotted line denotes a ghost. The expression is

$$
\begin{aligned}
\frac{T \log \mathcal{Z}}{V} \approx & -\frac{1}{2}(d-1) \mathcal{E}_{P} \log P^{2}+2 \oint_{\{P\}} \log \left(P^{2}+m^{2}\right) \\
& +\frac{1}{2} e^{2} \mathcal{E}_{\{P Q\}} \operatorname{Tr}\left[\gamma_{\mu} \frac{P-m}{P^{2}+m^{2}} \gamma_{\mu}\right. \\
& \left.\times \frac{\Phi-m}{Q^{2}+m^{2}} \frac{1}{(P+Q)^{2}}\right]
\end{aligned}
$$

where the trace is over Dirac indices. The sign $\approx$ is a reminder that the equality holds only in strict perturbation theory. The fermionic one-loop diagram has a pole in $\epsilon$, which is proportional to $m^{4}$. This pole is canceled by the vacuum counterterm $\Delta_{1} \mathcal{E}_{0}$. The two-loop diagram also has poles in $\epsilon$. The temperature-independent pole is canceled by the vacuum counterterm $\Delta_{2} \mathcal{E}_{0}$, while the temperaturedependent ones are canceled by the one-loop counterterm diagrams. The two-loop sum integral is briefly discussed in Appendix A. Our renormalization prescription is that the renormalized vacuum energy vanishes at the scale $\Lambda$. Thus the fermionic one-loop diagram and the two-loop diagram are given by their finite-temperature pieces [see Eqs. (A3) and (A23) in Appendix A]. After renormalization, the resulting expression in the low-temperature limit is

$$
\begin{aligned}
& \frac{T \log \mathcal{Z}}{V} \approx \frac{\pi^{2} T^{4}}{45}+\frac{4}{(2 \pi)^{3 / 2}} m^{3 / 2} T^{5 / 2} e^{-m / T} \\
& +\frac{e^{2}}{2(2 \pi)^{3}} m^{2} T^{2} e^{-2 m / T} \text {. }
\end{aligned}
$$

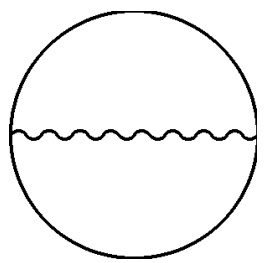

FIG. 2. Two-loop vacuum diagram in QED.

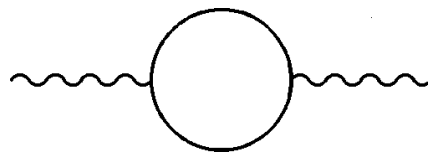

FIG. 3. One-loop polarization tensor in QED.

In the effective theory, all loop diagrams vanish with dimensional regularization, since there is no mass scale in the corresponding integrals. Hence, $\log \mathcal{Z}_{\text {eff }}$ vanishes identically in strict perturbation theory. The matching equation then implies

$$
f(\Lambda)=-\frac{\log \mathcal{Z}}{V}
$$

where the right hand side is given by Eq. (12).

\section{B. Field normalization constant}

The field normalization constants for $A_{i}$ and $A_{0}$ are obtained by reading off the coefficients of $\delta_{i j} k^{2}-k_{i} k_{j}$ and $k^{2}$ of $\Pi_{i j}^{(1)}(0, \mathbf{k})$ and $\Pi_{00}^{(1)}(0, \mathbf{k})$, where $\Pi_{\mu \nu}^{(1)}\left(k_{0}, \mathbf{k}\right)$ is the one-loop polarization tensor (C1) (see Fig. 3). These are given in Eqs. (C3) and (C4) in Appendix C, and we obtain

$$
\begin{aligned}
\bar{A}_{i}(\Lambda) \approx & \frac{1}{\sqrt{T}}\left[1+\frac{4}{3} e^{2} \mathcal{S}_{\{P\}} \frac{1}{\left(P^{2}+m^{2}\right)^{2}}\right]^{1 / 2} A_{i}, \\
\bar{A}_{0}(\Lambda) \approx & \frac{1}{\sqrt{T}}\left[1+\frac{1}{3} e^{2} \oint_{\{P\}} \frac{6}{\left(P^{2}+m^{2}\right)^{2}}\right. \\
& \left.-\frac{8 p_{0}^{2}}{\left(P^{2}+m^{2}\right)^{3}}\right]^{1 / 2} A_{0} .
\end{aligned}
$$

Equations (14) and (15) have poles in $\epsilon$ that are canceled by the wave function renormalization counterterm

$$
Z_{A}=1-\frac{e^{2}}{12 \pi^{2} \epsilon}
$$

After renormalization, we obtain

$$
\begin{aligned}
& \bar{A}_{i}(\Lambda) \approx \frac{1}{\sqrt{T}}\left[1+\frac{e^{2}}{24 \pi^{2}}\left(L-J_{2}\right)\right] A_{i}, \\
& \bar{A}_{0}(\Lambda) \approx \frac{1}{\sqrt{T}}\left[1+\frac{e^{2}}{24 \pi^{2}}\left(L-J_{2}-J_{3} m^{2} T^{-2}\right)\right] A_{0},
\end{aligned}
$$

where $L=\log \left(\Lambda^{2} / m^{2}\right)$ and the integrals $J_{n}$ are defined in Appendix A. Note the different normalizations of the fields $A_{0}$ and $A_{i}$. In the low-temperature limit, this reduces to

$$
\bar{A}_{i}(\Lambda) \rightarrow \frac{1}{\sqrt{T}}\left[1+\frac{e^{2}}{24 \pi^{2}}\left[L-2(2 \pi)^{1 / 2} m^{-1 / 2} T^{1 / 2} e^{-m / T}\right]\right] A_{i}
$$




$$
\bar{A}_{0}(\Lambda) \rightarrow \frac{1}{\sqrt{T}}\left[1+\frac{e^{2}}{24 \pi^{2}}\left[L-(2 \pi)^{1 / 2} m^{1 / 2} T^{-1 / 2} e^{-m / T}\right]\right] A_{0} .
$$

\section{Mass parameter}

The mass parameter can be determined in several ways. We determine it by demanding that the screening mass in strict perturbation theory be the same in QED and the effective theory. The screening mass $m_{s}$ is defined as the location of the pole in the propagator for spacelike momentum [19]:

$$
k^{2}+\Pi_{00}(0, \mathbf{k})=0, \quad k^{2}=-m_{s}^{2} .
$$

In the effective theory, we have

$$
k^{2}+M^{2}(\Lambda)+\Pi_{\text {eff }}(k, \Lambda)=0, \quad k^{2}=-m_{s}^{2},
$$

where $\Pi_{\text {eff }}(k, \Lambda)$ is the self-energy of $\bar{A}_{0}$ in the effective theory. We can expand the self-energy function $\Pi_{00}(0, \mathbf{k})$ in powers of the external momentum $\mathbf{k}$. To second order in the loop expansion, the solution to Eq. (21) for the screening mass is [21]

$$
m_{s}^{2} \approx\left[1-\Pi_{00}^{(1)^{\prime}}(0,0)\right] \Pi_{00}^{(1)}(0,0)+\Pi_{00}^{(2)}(0,0) .
$$

Here $\Pi_{00}^{(n)}$ denotes the $n$th order contribution to the static polarization tensor in the loop expansion, and the prime denotes differentiation with respect to $k^{2}$. The self-energy function $\Pi_{\text {eff }}(k, \Lambda)$ can also be expanded in a Taylor series around $\mathbf{k}=\mathbf{0}$. The corresponding loop integrals are evaluated at $\mathbf{k}=\mathbf{0}$ and since there is no other mass scale, the selfenergy function $\Pi_{\text {eff }}(0, \Lambda)$ vanishes identically in strict perturbation theory. Thus the matching condition reduces to

$$
M^{2}(\Lambda) \approx m_{s}^{2} .
$$

The one-loop contribution to $\Pi_{00}(0,0)$ is given by the first term in Eq. (C4), while $\Pi_{00}^{\prime}(0,0)$ is given by the second term in Eq. (C4). The two-loop contribution to $\Pi_{00}(0,0)$ is given by Eq. (A27), and in Appendix B, we explain how one can obtain the expression for it from the two-loop contribution to the free energy. In the low-temperature limit, the mass parameter becomes

$$
\begin{aligned}
M^{2}(\Lambda)= & \frac{4 e^{2}}{(2 \pi)^{3 / 2}} m^{3 / 2} T^{1 / 2} e^{-m / T}-\frac{4 e^{4}}{3(2 \pi)^{7 / 2}} m^{3 / 2} T^{1 / 2} L e^{-m / T} \\
& +\frac{10 e^{4}}{3(2 \pi)^{3}} m^{2} e^{-2 m / T} .
\end{aligned}
$$

Using the renormalization group equation for the running gauge coupling,

$$
\mu \frac{d e^{2}}{d \mu}=\frac{e^{4}}{6 \pi^{2}}
$$

we see that the mass parameter is independent of the renormalization scale $\mu$ to order $e^{4}$.

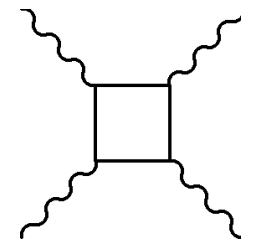

FIG. 4. Four-point function with external timelike photons at one loop in QED.

\section{Coupling constants}

The one-loop Feynman diagram for the four-point function $\Gamma^{(4)}$ with external timelike photons is shown in Fig 4. The matching equation is simply [21]

$$
\lambda_{3}(\Lambda)=T \Gamma^{(4)}(0,0,0,0),
$$

where the arguments indicate that the loop diagram is to be evaluated at zero external momenta The expression for the diagram is

$$
\begin{aligned}
& \Gamma^{(4)}(0,0,0,0)=6 e^{2} \oint_{\{P\}} \operatorname{Tr}\left[\gamma_{0} \frac{\boldsymbol{P}-m}{P^{2}+m^{2}} \gamma_{0} \frac{\boldsymbol{P}-m}{P^{2}+m^{2}} \gamma_{0}\right. \\
& \left.\times \frac{p-m}{P^{2}+m^{2}} \gamma_{0} \frac{p-m}{P^{2}+m^{2}}\right] \\
& =24 e^{2} \oint_{\{P\}}\left[m^{4}+2 m^{2} P^{2}-8 m^{2} p_{0}^{2}+P^{4}\right. \\
& \left.-8 p_{0}^{2} P^{2}+8 p_{0}^{4}\right] \frac{1}{\left(P^{2}+m^{2}\right)^{4}} .
\end{aligned}
$$

The particular combination of sum integrals in Eq. (28) is finite with dimensional regularization and we obtain

$$
\lambda_{3}(\Lambda)=-\frac{8 e^{4}}{(2 \pi)^{2}} J_{4} m^{4} T^{-3} .
$$

The fact that this coefficient vanishes identically at zero temperature, simply reflects the gauge invariance of QED. In the low-temperature limit, Eq. (29) reduces to

$$
\lambda_{3}(\Lambda)=-\frac{4 e^{4}}{(2 \pi)^{3 / 2}} m^{3 / 2} T^{-1 / 2} e^{-m / T} .
$$

The coupling constant $a_{3}$ is not affected by the field redefinition Eqs. (17) to leading order in $e$, so its value is given directly by the coefficient of the last term in Eq. (C3):

$$
\begin{aligned}
a_{3}(\Lambda) & =\frac{8}{15} e^{2} \oiint_{\{P\}} \frac{1}{\left(P^{2}+m^{2}\right)^{3}} \\
& =\frac{e^{2}}{60 \pi^{2} m^{2}}\left[1-m^{2} T^{-2} J_{3}\right] .
\end{aligned}
$$

In the low-temperature limit, this reduces to 


$$
a_{3}(\Lambda)=\frac{e^{2}}{60 \pi^{2} m^{2}}\left[1-(2 \pi)^{1 / 2} m^{1 / 2} T^{-1 / 2} e^{-m / T}\right] .
$$

The first term is the standard zero-temperature Uehling term, while the second is a new thermal correction.

\section{FREE ENERGY AND DEBYE MASS}

In this section, we use the effective Lagrangian Eq. (5) to calculate the free energy to order $e^{3}$ and the Debye mass to order $e^{5}$, respectively. In order to take electric screening properly into account, we must include the effects of the mass term $M$ to all orders in perturbation theory. Thus the Lagrangian is split accordingly:

$$
\begin{aligned}
\mathcal{L}_{\text {eff }}^{\text {free }}= & \frac{1}{4} F_{i j} F_{i j}+\frac{1}{2}\left(\partial_{i} \bar{A}_{0}\right)^{2}+\frac{1}{2} M^{2}(\Lambda) \bar{A}_{0}^{2}+\left(\partial_{\mu} \bar{\eta}\right)\left(\partial_{\mu} \eta\right) \\
& +\mathcal{L}_{\mathrm{GF}} \\
\mathcal{L}_{\text {eff }}^{\text {int }}= & \frac{1}{4} a_{3}(\Lambda) F_{i j} \nabla^{2} F_{i j}+\frac{\lambda_{3}(\Lambda)}{24} \bar{A}_{0}^{4}+\delta \mathcal{L}_{\text {eff }} .
\end{aligned}
$$

The $e^{3}$ contribution to the free energy is given by a simple one-loop calculation in the effective theory:

$$
\begin{aligned}
\frac{T \log \mathcal{Z}_{\text {eff }}}{V}= & -\frac{1}{2} T \int_{p} \log \left(p^{2}+M^{2}\right)-\frac{1}{2}(d-2) T \\
& \times \int_{p} \log p^{2}+\frac{1}{2} a_{3}(\Lambda)(d-2) \int_{p} p^{2} .
\end{aligned}
$$

The total free energy is then given by

$$
\mathcal{F}=f(\Lambda) T-\frac{T \log \mathcal{Z}_{\text {eff }}}{V},
$$

where $f(\Lambda)$ is given by Eq. (12). Using Eq. (B3) in Appendix $\mathrm{B}$ and the expression for the mass parameter $M(\Lambda)$ to leading order, Eq. (36) reduces to

$$
\frac{T \log \mathcal{Z}_{\text {eff }}}{V}=\frac{4 e^{3} m^{9 / 4} T^{7 / 4}}{3(2 \pi)^{13 / 4}} e^{-3 m / 2 T} .
$$

The total free energy density is minus the sum of Eqs. (12) and (38):

$$
\begin{aligned}
\mathcal{F}= & -\frac{\pi^{2} T^{4}}{45}-\frac{4}{(2 \pi)^{3 / 2}} m^{3 / 2} T^{5 / 2} e^{-m / T}-\frac{e^{2}}{2(2 \pi)^{3}} m^{2} T^{2} e^{-2 m / T} \\
& -\frac{4 e^{3} m^{9 / 4} T^{7 / 4}}{3(2 \pi)^{13 / 4}} e^{-3 m / 2 T} .
\end{aligned}
$$

Equation (39) is in agreement with the result first obtained by Gell-Mann and Bruc̈kner [6] in nonrelativistic QED. Note the term that is nonanalytic in $e^{2}$. It arises from the summation of an infinite number of infrared divergent loops (ring diagrams).
The Debye mass $m_{\mathrm{D}}$ is given by the location of the pole in the propagator

$$
k^{2}+M^{2}(\Lambda)+\Pi_{\mathrm{eff}}(\mathbf{k}, \Lambda)=0, \quad k=i m_{\mathrm{D}},
$$

where $\Pi_{\text {eff }}(\mathbf{k}, \Lambda)$ denotes the self-energy function of $\bar{A}_{0}$. To leading order in $e$, the solution to Eq. (40) is simply $m_{\mathrm{D}}^{2}$ $=M^{2}(\Lambda)$. The one-loop approximation to the self-energy is

$$
\Pi_{\mathrm{eff}}^{(1)}(\mathbf{k}, \Lambda)=\frac{1}{2} \lambda_{3}(\Lambda) \int_{p} \frac{1}{p^{2}+M^{2}} .
$$

$\Pi_{\text {eff }}^{(1)}(\mathbf{k}, \Lambda)$ is independent of the external momentum, so the solution to Eq. (40) is simply

$$
m_{\mathrm{D}}^{2}=M^{2}(\Lambda)+\Pi_{\mathrm{eff}}^{(1)}(\mathbf{k}, \Lambda) .
$$

Using Eq. (B3) and expanding the mass parameter $M(\Lambda)$ in powers of $e$, we obtain the Debye mass through order $e^{5}$ :

$$
\begin{aligned}
m_{\mathrm{D}}^{2}= & \frac{4 e^{2}}{(2 \pi)^{3 / 2}} m^{3 / 2} T^{1 / 2} e^{-m / T}-\frac{4 e^{4}}{3(2 \pi)^{7 / 2}} m^{3 / 2} T^{1 / 2} L e^{-m / T} \\
& +\frac{10 e^{4}}{3(2 \pi)^{3}} m^{2} e^{-2 m / T}+\frac{2 e^{5}}{(2 \pi)^{13 / 4}} m^{9 / 4} T^{-1 / 4} e^{-3 m / 2 T} .
\end{aligned}
$$

Equation (43) is the main result of the present paper. Using the renormalization group equation (26) for the running gauge coupling, we see that the Debye mass is independent of the renormalization scale $\Lambda$ up to corrections of order $e^{6}$. Note also that there is no term proportional to $e^{3}$ in the expression for the Debye mass. The reason is that there are no bosonic propagators in the one-loop self-energy graph in QED and fermions need no resummation, since their Matsubara frequencies are never zero.

\section{SUMMARY}

In the present work, we have studied QED at low temperature. We have constructed an effective field theory in three dimensions that is valid at distances $R \gg 1 / T$ by integrating out the electron field and the nonzero Matsubara modes. Three-dimensional field theory was used to calculate the pressure to order $e^{3}$ and the Debye mass to order $e^{5}$. The pressure and the Debye mass can be calculated either by resummation or by effective field theory. Not only does effective field theory simplify these calculations, it also unravels the contributions to physical quantities from different momentum scales.

\section{ACKNOWLEDGMENTS}

This work was supported by the Stichting voor Fundamenteel Onderzoek der Materie (FOM), which is supported by the Nederlandse Organisatie voor Wetenschapplijk Onderzoek (NWO). 


\section{APPENDIX A: SUM INTEGRALS}

In the imaginary-time formalism for thermal field theory, the 4-momentum $P=\left(p_{0}, \mathbf{p}\right)$ is Euclidean with $P^{2}=p_{0}^{2}+\mathbf{p}^{2}$. The Euclidean energy $p_{0}$ has discrete values: $p_{0}=2 n \pi T$ for bosons and $p_{0}=(2 n+1) \pi T$ for fermions, where $n$ is an integer. Loop diagrams involve sums over $p_{0}$ and integrals over $\mathbf{p}$. With dimensional regularization, the integral is generalized to $d=3-2 \epsilon$ spatial dimensions. We define the dimensionally regularized sum integral by

$$
\begin{gathered}
\mathcal{S}_{P} \equiv\left(\frac{e^{\gamma} \mu^{2}}{4 \pi}\right)^{\epsilon} T \sum_{p_{0}=2 n \pi T} \int \frac{d^{3-2 \epsilon} p}{(2 \pi)^{3-2 \epsilon}}, \\
\oint_{\{P\}} \equiv\left(\frac{e^{\gamma} \mu^{2}}{4 \pi}\right)^{\epsilon} T_{p_{0}=(2 n+1) \pi T} \sum \int \frac{d^{3-2 \epsilon} p}{(2 \pi)^{3-2 \epsilon}},
\end{gathered}
$$

where $\mu$ is an arbitrary momentum scale. The factor $\left(e^{\gamma / 4 \pi}\right)^{\epsilon}$ is introduced so that, after minimal subtraction of the poles in $\epsilon$ due to ultraviolet divergences, $\mu$ coincides with the renormalization scale of the modified minimal subtraction $(\overline{\mathrm{MS}})$ renormalization scheme.

\section{One-loop sum integrals}

The specific one-loop fermionic sum integrals needed are

$$
\begin{aligned}
\oint_{\{P\}} \log \left(P^{2}+m^{2}\right)= & \frac{1}{(4 \pi)^{2}}\left(\frac{\mu}{m}\right)^{2 \epsilon} \\
& \times\left[-\frac{e^{\gamma \epsilon} \Gamma(1+\epsilon)}{\epsilon(1-\epsilon)(2-\epsilon)} m^{4}+J_{0} T^{4}\right]
\end{aligned}
$$

$$
\begin{aligned}
\mathcal{X}_{\{P\}} \frac{1}{P^{2}+m^{2}}= & \frac{1}{(4 \pi)^{2}}\left(\frac{\mu}{m}\right)^{2 \epsilon} \\
& \times\left[-\frac{e^{\gamma \epsilon} \Gamma(1+\epsilon)}{\epsilon(1-\epsilon)} m^{2}-J_{1} T^{2}\right],
\end{aligned}
$$

$$
\oint_{\{P\}} \frac{1}{\left(P^{2}+m^{2}\right)^{2}}=\frac{1}{(4 \pi)^{2}}\left(\frac{\mu}{m}\right)^{2 \epsilon}\left[\frac{e^{\gamma \epsilon} \Gamma(1+\epsilon)}{\epsilon}-J_{2}\right],
$$

$$
\begin{aligned}
\sum_{\{P\}} \frac{1}{\left(P^{2}+m^{2}\right)^{3}}= & \frac{1}{2(4 \pi)^{2}}\left(\frac{\mu}{m}\right)^{2 \epsilon} \\
& \times\left[e^{\gamma \epsilon} \Gamma(1+\epsilon) m^{-2}-J_{3} T^{-2}\right],
\end{aligned}
$$

$$
\begin{aligned}
\oint_{\{P\}} \frac{1}{\left(P^{2}+m^{2}\right)^{4}}= & \frac{1}{6(4 \pi)^{2}}\left(\frac{\mu}{m}\right)^{2 \epsilon}\left[e^{\gamma \epsilon} \Gamma(1+\epsilon)\right. \\
& \left.\times(1+\epsilon) m^{-4}-J_{4} T^{-4}\right]
\end{aligned}
$$

$$
\begin{aligned}
\sum_{\{P\}} \frac{p_{i} p_{j}}{\left(P^{2}+m^{2}\right)^{2}}= & \frac{\delta_{i j}}{2(4 \pi)^{2}}\left(\frac{\mu}{m}\right)^{2 \epsilon} \\
& \times\left[-\frac{e^{\gamma \epsilon} \Gamma(1+\epsilon)}{\epsilon(1-\epsilon)} m^{2}-J_{1} T^{2}\right],
\end{aligned}
$$$$
\oint_{\{P\}} \frac{p_{0}^{2}}{\left(P^{2}+m^{2}\right)^{2}}=\frac{1}{2(4 \pi)^{2}}\left(\frac{\mu}{m}\right)^{2 \epsilon}\left[-\frac{e^{\gamma \epsilon} \Gamma(1+\epsilon)}{\epsilon(1-\epsilon)} m^{2}\right.
$$$$
\left.+(d-2) J_{1} T^{2}+2 J_{2} m^{2}\right]
$$

$$
\begin{aligned}
\mathcal{E}_{\{P\}} \frac{p_{0}^{2}}{\left(P^{2}+m^{2}\right)^{3}}= & \frac{1}{4(4 \pi)^{2}}\left(\frac{\mu}{m}\right)^{2 \epsilon}\left[\frac{e^{\gamma \epsilon} \Gamma(1+\epsilon)}{\epsilon}\right. \\
& \left.+(d-4) J_{2}+2 J_{3} m^{2} T^{-2}\right]
\end{aligned}
$$

$$
\begin{aligned}
\mathcal{E}_{\{P\}} \frac{p_{0}^{2}}{\left(P^{2}+m^{2}\right)^{4}}= & \frac{1}{12(4 \pi)^{2}}\left(\frac{\mu}{m}\right)^{2 \epsilon}\left[e^{\gamma \epsilon} \Gamma(1+\epsilon) m^{-2}\right. \\
& \left.+(d-6) J_{3} T^{-2}+2 J_{4} m^{2} T^{-4}\right],
\end{aligned}
$$

$$
\begin{aligned}
\oint_{\{P\}} \frac{p_{0}^{2} p_{i} p_{j}}{\left(P^{2}+m^{2}\right)^{4}}= & \frac{\delta_{i j}}{24(4 \pi)^{2}}\left(\frac{\mu}{m}\right)^{2 \epsilon}\left[\frac{e^{\gamma \epsilon} \Gamma(1+\epsilon)}{\epsilon}\right. \\
& \left.+(d-4) J_{2}+2 J_{3} m^{2} T^{-2}\right],
\end{aligned}
$$

The integrals $J_{n}(\beta m)$ can be expressed as integrals involving the Fermi-Dirac distribution function:

$$
\begin{aligned}
J_{n}(\beta m)= & \frac{4 e^{\gamma \epsilon} \Gamma\left(\frac{1}{2}\right)}{\Gamma\left(\frac{5}{2}-n-\epsilon\right)} \beta^{4-2 n} m^{2 \epsilon} \\
& \times \int_{0}^{\infty} d k \frac{k^{4-2 n-2 \epsilon}}{\left(k^{2}+m^{2}\right)^{1 / 2}} \frac{1}{e^{\beta\left(k^{2}+m^{2}\right)^{1 / 2}}+1} .
\end{aligned}
$$

These integrals are functions of $\beta m$ only and satisfy the recursion relation

$$
m \frac{\partial}{\partial m} J_{n}(\beta m)=2 \epsilon J_{n}(\beta m)-2(\beta m)^{2} J_{n+1}(\beta m) .
$$

We need the integrals $J_{n}$ for $\epsilon=0$. In the low-temperature limit, these integrals reduce to

$$
\begin{aligned}
& J_{0} \rightarrow 8(2 \pi)^{1 / 2} m^{3 / 2} T^{-3 / 2} e^{-m / T}, \\
& J_{1} \rightarrow 4(2 \pi)^{1 / 2} m^{1 / 2} T^{-1 / 2} e^{-m / T}, \\
& J_{2} \rightarrow 2(2 \pi)^{1 / 2} m^{-1 / 2} T^{1 / 2} e^{-m / T},
\end{aligned}
$$




$$
\begin{aligned}
& J_{3} \rightarrow(2 \pi)^{1 / 2} m^{-3 / 2} T^{3 / 2} e^{-m / T}, \\
& J_{4} \rightarrow \frac{1}{2}(2 \pi)^{1 / 2} m^{-5 / 2} T^{5 / 2} e^{-m / T} .
\end{aligned}
$$

Note that the integrals $J_{3}$ and $J_{4}$ need subtractions to remove power infrared divergences:

$$
\begin{aligned}
J_{3}(\beta m)= & -2 T^{2} \int_{0}^{\infty} d k \frac{1}{k^{2}}\left[\frac{1}{\left(k^{2}+m^{2}\right)^{1 / 2}} \frac{1}{e^{\beta\left(k^{2}+m^{2}\right)^{1 / 2}}+1}\right. \\
& \left.-\frac{1}{m} \frac{1}{e^{\beta\left(k^{2}+m^{2}\right)^{1 / 2}}+1}\right], \\
J_{4}(\beta m)= & 3 T^{4} \int_{0}^{\infty} d k \frac{1}{k^{4}}\left[\frac{1}{\left(k^{2}+m^{2}\right)^{1 / 2}} \frac{1}{e^{\beta\left(k^{2}+m^{2}\right)^{1 / 2}+1}}\right. \\
& -\frac{1}{m} \frac{1}{\left.e^{\beta\left(k^{2}+m^{2}\right)^{1 / 2}+1}+\frac{k^{2}}{2 m^{3}} \frac{1}{e^{\beta\left(k^{2}+m^{2}\right)^{1 / 2}}+1}\right] .}
\end{aligned}
$$

The specific one-loop bosonic sum integral needed is

$$
\sum_{P} \log \left(P^{2}\right)=-\frac{\pi^{2} T^{4}}{45}[1+O(\epsilon)] .
$$

\section{Two-loop sum integral}

We also need the value of the two-loop diagram in Eq. (11). The two-loop sum integral with nonzero chemical $\mu$ potential was calculated in e.g. Refs. [7,8,22]. There are $T$-dependent and $\mu$-dependent infinities in addition to the usual vacuum infinities. These are canceled by the corresponding infinities arising from the one-loop counterterm diagrams. The vacuum infinity is canceled by a vacuum counterterm in the usual way. If we demand that the twoloop contribution to the vacuum energy vanishes at the scale $\Lambda$, the diagram is given by its finite-temperature piece. The final result after renormalization is then [8]

$$
\begin{aligned}
& \frac{1}{2} e^{2} \mathcal{S}_{\{P Q\}} \operatorname{Tr}\left[\gamma_{\mu} \frac{P-m}{P^{2}+m^{2}} \gamma_{\mu} \frac{\Phi-m}{Q^{2}+m^{2}} \frac{1}{(P+Q)^{2}}\right] \\
&=-\frac{e^{2}}{12 \pi^{2}} T^{2} \int_{0}^{\infty} d p \frac{p^{2}}{E_{p}}\left[n_{p}^{+}+n_{p}^{-}\right] \\
&-\frac{e^{2}}{16 \pi^{4}} \int_{0}^{\infty} d p d q \frac{p^{2} q^{2}}{E_{p} E_{q}}\left[\left(2+\frac{m^{2}}{p q} \log \frac{E_{p} E_{q}-m^{2}-p q}{E_{p} E_{q}-m^{2}+p q}\right)\right. \\
& \times\left(n_{p}^{-} n_{q}^{-}+n_{p}^{+} n_{q}^{+}\right)+\left(2+\frac{m^{2}}{p q} \log \frac{E_{p} E_{q}+m^{2}+p q}{E_{p} E_{q}+m^{2}-p q}\right) \\
&\left.\times\left(n_{p}^{-} n_{q}^{+}+n_{p}^{+} n_{q}^{-}\right)\right],
\end{aligned}
$$

where $E_{p}=\sqrt{p^{2}+m^{2}}$ and

$$
n_{p}^{ \pm}=\frac{1}{e^{\beta\left(E_{p} \pm \mu\right)}+1} .
$$

In the low-temperature limit, this reduces to

$$
\begin{gathered}
\frac{1}{2} e^{2} \mathcal{\Psi}_{\{P Q\}} \operatorname{Tr}\left[\gamma_{\mu} \frac{P-m}{P^{2}+m^{2}} \gamma_{\mu} \frac{\Phi-m}{Q^{2}+m^{2}} \frac{1}{(P+Q)^{2}}\right] \\
\rightarrow \frac{e^{2}}{2(2 \pi)^{3}} m^{2} T^{2} e^{2(\mu-m) / T} .
\end{gathered}
$$

Equation (A23) gives the two-loop contribution to the pressure $\mathcal{P}$. By applying the formula [22]

$$
\Pi_{00}(0,0)=e^{2} \frac{\partial^{2} \mathcal{P}^{2}}{\partial \mu^{2}}
$$

to Eq. (A25), we obtain the two-loop contribution to the photon polarization tensor at zero external momentum and vanishing chemical potential:

$$
\Pi_{00}^{(2)}(0,0)=\frac{2 e^{4}}{(2 \pi)^{3}} m^{2} e^{-2 m / T} .
$$

\section{APPENDIX B: INTEGRALS}

Dimensional regularization can be used to regularize both the ultraviolet divergences and infrared divergences in 3-dimensional integrals over momenta. The spatial dimension is generalized to $d=3-2 \epsilon$ dimensions. Integrals are evaluated at a value of $d$ for which they converge and then analytically continued to $d=3$. We use the integration measure

$$
\int_{p} \equiv\left(\frac{e^{\gamma} \mu^{2}}{4 \pi}\right)^{\epsilon} \int \frac{d^{3-2 \epsilon} p}{(2 \pi)^{3-2 \epsilon}}
$$

where $\mu$ is an arbitrary momentum scale. The factor $\left(e^{\gamma / 4 \pi}\right)^{\epsilon}$ is introduced so that, after minimal subtraction of the poles in $\epsilon$ due to ultraviolet divergences, $\mu$ coincides with the renormalization scale of the $\overline{\mathrm{MS}}$ renormalization scheme.

The one-loop integrals required are

$$
\begin{aligned}
\int_{p} \log \left(p^{2}+m^{2}\right) & =-\frac{m^{3}}{6 \pi}[1+O(\epsilon)], \\
\int_{p} \frac{1}{p^{2}+m^{2}} & =-\frac{m}{4 \pi}[1+O(\epsilon)] .
\end{aligned}
$$

\section{APPENDIX C: POLARIZATION TENSOR}

In this appendix, we calculate the polarization tensor $\Pi_{\mu \nu}\left(\omega_{n}, \mathbf{k}\right)$ to one loop. The Feynman diagram is shown in Fig. 3 and the expression for it is 


$$
\Pi_{\mu \nu}^{(1)}\left(k_{0}, \mathbf{k}\right)=e^{2} \oint_{\{P\}} \operatorname{Tr}\left[\gamma_{\mu} \frac{\boldsymbol{P}-m}{P^{2}+m^{2}} \gamma_{\nu} \frac{(\boldsymbol{P}+\mathbb{K})-m}{(P+K)^{2}+m^{2}}\right] .
$$

Taking the Dirac trace, and using a Feynman parameter $y$, this can be written as

$$
\begin{aligned}
\Pi_{\mu \nu}^{(1)}\left(k_{0}, \mathbf{k}\right)= & e^{2} \int_{0}^{1} d y \oint_{\{P\}}\left[\frac{8 p_{\mu} p_{\nu}}{\left[P^{2}+m^{2}+K^{2} y(1-y)\right]^{2}}\right. \\
& -\frac{4 \delta_{\mu \nu}}{\left[P^{2}+m^{2}+K^{2} y(1-y)\right]} \\
& \left.+\frac{8 y(1-y)\left(\delta_{\mu \nu} K^{2}-k_{\mu} k_{\nu}\right)}{\left[P^{2}+m^{2}+K^{2} y(1-y)\right]^{2}}\right]
\end{aligned}
$$

First consider $\Pi_{i j}^{(1)}(0, \mathbf{k})$. By virtue of Eqs. (A4) and (A8), the first two terms cancel identically. The remaining term is expanded to second order in a Taylor series around $\mathbf{k}=0$. Integrating over $y$, we obtain

$$
\begin{aligned}
\Pi_{i j}^{(1)}(0, \mathbf{k})= & \frac{4}{3} e^{2}\left(\delta_{i j} k^{2}-k_{i} k_{j}\right) \oint_{\{P\}} \frac{1}{\left(P^{2}+m^{2}\right)^{2}} \\
& -\frac{8}{15} e^{2} k^{2}\left(\delta_{i j} k^{2}-k_{i} k_{j}\right) \oint_{\{P\}} \frac{1}{\left(P^{2}+m^{2}\right)^{3}} .
\end{aligned}
$$

The first term gives the one-loop correction to the field normalization constants, while the second gives the coefficient of the Uehling term. The fact that $\Pi_{i j}^{(1)}(0, \mathbf{k})$ vanishes in the limit $\mathbf{k} \rightarrow \mathbf{0}$ reflects the fact that there is no screening of static magnetic fields in QED.

Consider next $\Pi_{00}^{(1)}(0, \mathbf{k})$. Expanding to second order in the external momentum $\mathbf{k}$ and integrating over $y$ yields

$$
\begin{aligned}
\Pi_{00}^{(1)}(0, \mathbf{k})= & 4 e^{2} \oint_{\{P\}}\left[\frac{2 p_{0}^{2}}{\left(P^{2}+m^{2}\right)^{2}}-\frac{1}{P^{2}+m^{2}}\right] \\
& +\frac{1}{3} e^{2} k^{2} \oint_{\{P\}}\left[\frac{6}{\left(P^{2}+m^{2}\right)^{2}}-\frac{8 p_{0}^{2}}{\left(P^{2}+m^{2}\right)^{3}}\right] .
\end{aligned}
$$

The first term gives the one-loop expression for the mass parameter (which coincides with the one-loop expression for the Debye mass), while the second term gives the one-loop correction to the field normalization constant.
[1] G. Barton, Ann. Phys. (N.Y.) 205, 49 (1991).

[2] X. Kong and F. Ravndal, Nucl. Phys. B526, 627 (1998).

[3] E. Braaten and Y. Jia, Phys. Rev. D 63, 096009 (2001).

[4] G. P. Lepage, in From Actions to Answers, Proceedings of the Advanced Study Institute in Elementary Particle Physics, Boulder, Colorado, 1989, edited by T. De-Grand and D. Toussaint (World Scientific, Singapore, 1989).

[5] D. B. Kaplan, Lectures given at 7th Summer School in Nuclear Physics Symmetries, Seattle, WA, 1995, nucl-th/9506035.

[6] M. Gell-Mann and K. A. Brückner, Phys. Rev. 106, 364 (1957).

[7] J. I. Kapusta, Nucl. Phys. B148, 461 (1979).

[8] T. Toimela, Int. J. Theor. Phys. 24, 901 (1985); 26, 1021(E) (1987).

[9] R. M. Woloshyn, Phys. Rev. D 27, 1393 (1983).

[10] R. Tarrach, Phys. Lett. 133B, 259 (1983).

[11] J. I. Latorre, P. Pascual, and R. Tarrach, Nucl. Phys. B437, 60
(1995).

[12] P. Elmfors, D. Persson, and B.-S. Skagerstam, Astropart. Phys. 2, 299 (1994).

[13] H. Gies, Phys. Rev. D 61, 085021 (2000); hep-ph/0010287.

[14] E. A. Uehling, Phys. Rev. 48, 55 (1935).

[15] H. Euler, Ann. Phys. (Leipzig) 26, 398 (1936); W. Heisenberg and H. Euler, Z. Phys. 98, 714 (1936).

[16] D. A. Dicus, C. Kao, and W. W. Repko, Phys. Rev. D 57, 2443 (1998).

[17] N. P. Landsman, Nucl. Phys. B322, 498 (1989).

[18] P. Ginsparg, Nucl. Phys. B170, 388 (1980).

[19] E. Braaten and A. Nieto, Phys. Rev. D 51, 6990 (1995).

[20] K. Kajantie, M. Laine, K. Rummukainen, and M. Shaposhnikov, Nucl. Phys. B458, 90 (1996).

[21] J. O. Andersen, Phys. Rev. D 53, 7286 (1996).

[22] J. I. Kapusta, Finite Temperature Field Theory (Cambridge University Press, Cambridge, England, 1989). 